\title{
H7. Endocrine Response of Brain Tumor.
}

\author{
K. SAWAdA and T. WADA \\ Dept. of Surg., Faculty of Med., Tohoku Univ.
}

About 30 cases of brain tumor, the daily serum antidiuretic substance (ADS) and total 17-hydroxycorticosteroids (17-OHCS) of urine during 24 hours were measured, and the endocrine responces to pre- and post-operation were observed under the normo- and hypothermic anesthesia by these factors.

Though result showed us much variation in each case, as general tendency, 17-OHCS and ADS showed great increasing for a few days after normothermic operation.

But in the hypothermic anesthesia the tendency about the excretion of 17-OHCS was not constant, and besides ADS was relatively decreasing during 2 days after the operation.

We expect that hypothermic method may inhibit the brain edema after the procedure by suppression for the secretion of antidiuretic hormone.

\section{H8. Study on the Surgery of Craniopharyngioma.}

\author{
T. Wada, J. Suzuki, H. Seno, A. Narita, K. Sawada, \\ S. Kowada, S. Saso and N. Harata \\ Dept. of Surg., Faculty of Med., Tohoku Univ.
}

28 cases of craniopharyngioma; male 17 cases, female 11 cases, were experienced in our clinic. The most important point to diagnose craniopharyngioma is the finding of calcification shadow on X-photography, and we recognized it on about $80 \%$ of our cases. This percentage was the most in all kind of brain tumors.

This calcification which deposited on hyalinsubstance surrounded with epithelial cell, is seen as a fleecy shadow in usual, but sometimes shows an archshadow when calcium deposited on the wall of cyst.

Their localization is generally on the circumference of sella turcica but we have to take care of the fact that the calcium shadow is seen in distant part when tumor is enormous.

The second point is the visual disturbance which is seen almost every cases. Especially the change of papilla and of visual field are necessary to decide the localization of tumor and its deviation to right or left.

By synthesis with the characterisity of calcium shadow, localization, pneumoventriculography, nervous symptom and the finding of visual field, we can recognize the localization, size and deviation of tumor quite sure. 
At this point of view, we can classify the localization, namely 1) tumor located in ront of optic chiasma, 2) in sella turcica, 3) at temporal side, 4) invaded into third ventricle, 5) developed on the backward of basilar region and up to subtentorium and 6) extradural development.

In the past, Dandy's Approach by frontal trepanation was often applied for every cases. But we think that such method is only applied for cases of which classified 1) and 2), and for others it is better to apply the temporal trepanation especially Valladares's Approach. According to this method, as temporal muscle was intersected, operation field is kept not so deep and in some cases, which depend on the characteristics of tumors, the total resection is possible. Recently we succeeded it by this method.

The sum of the matter, we have to consider the method of operation, after the localization of tumor was decided by the full considering of symptom.

\title{
H9. Positive Contrast Ventriculography.
}

\author{
N. Hoshino \\ Dept. of Surg., Hiroshima Univ. Med. School
}

There can be no objection to admit the high reliability of positive contrast ventriculography in the diagnosis of suspectable brain tumor. Undesirable sideeffects, however, have made almost all neurosurgeons to abandon this diagnostic tool. In my experiences of about 350 cases of ventriculography using MOLJODOL (identical to lipiodol), increase in body temperature above $38.5^{\circ} \mathrm{C}$ was seen in about a half of cases, one fifth of which being hyperthermia above $39.5^{\circ} \mathrm{C}$. Aggravation of headache and vomiting was also complained in about $50 \%$. Occasionally serious complications, such as disturbances in consciousness, convulsive seizures or respiratory arrest were caused, and the direct mortality reached to $4.7 \%$. These ill-effects were most conspicuous in brain tumors with occlusive hydrocephalus, i.e. in diseases most necessitating the positive contrast ventriculography.

Recently I used to use MYODIL (identical to pantopaque) as the contrast dye for ventriculography. It is true that intraventricular injection of MYODIL sometimes caused increase in body temperature, but never reached to hyperthermia. Hadache and vomiting were minimum. There were no cases of disturbances in consciousness and no patient was lost. In particular, if the continuous ventricular tapping was combined with the injection of dye, hardly any side-effects could be seen.

In summary, positive contrast ventriculography using MYODIL should be adopted without hesitation if other diagnostic procedures failed to reveal the exact and precise localizing diagnosis. 